\title{
Turbo Packet Combining for Broadband MIMO Relay Communication
}

\author{
Tarik Ait-Idir ${ }^{\ddagger *}$, Houda Chafnaji ${ }^{\ddagger *}$, Halim Yanikomeroglu ${ }^{+}$, and Samir Saoudi* \\ ${ }^{\ddagger}$ Communications Systems Department, INPT, Madinat Al Irfane, Rabat, Morocco \\ *INSTITUT TELECOM/ TELECOM Bretagne, Signal and Communications Department, CS 83818, 29238 Brest Cedex, France. \\ + Broadband Communications and Wireless Systems (BCWS) Centre, \\ Department of Systems and Computer Engineering, Carleton University, Ottawa, Canada \\ Emails: aitidir@ieee.org, houda.chafnaji,samir.saoudi@telecom-bretagne.eu, halim@sce.carleton.ca
}

\begin{abstract}
In this paper, we provide a survey of cooperative automatic repeat request (ARQ) protocols for multi-relay systems operating over multiple-input-multiple-output (MIMO) broadband channels. We study three main operating modes: amplify-and-forward (AF), decode-and-forward (DF), and positive/negative acknowledgment (ACK/NACK)-aided DF. We describe efficient minimum mean square error (MMSE) frequency domain equalization (FDE) iterative turbo packet combining techniques for cooperative $A R Q$, and show that they outperform conventional log-likelihood ratio (LLR)-based turbo combining.
\end{abstract}

Index Terms-Cooperative relaying, multiple-antenna systems, turbo equalization, packet combining.

\section{INTRODUCTION}

Automatic repeat request (ARQ) and forward error correction (FEC) are two major techniques for increasing the diversity gain of wireless fading channels [1], [2]. In slow fading wireless environments, ARQ mechanisms generally have limited performance due to the long-term static dynamic of the ARQ fading channel where multiple ARQ rounds see the same channel realizations. To overcome this limitation, cooperative relay communication has been introduced [3]. It presents an efficient technique for building up a virtual short-term static ARQ channel where virtual ARQ rounds see independent channel realizations. This is achieved by using multiple relays which act as packet re-transmitters. In cooperative relay communications, the source first broadcasts the information packet to the destination. In case of erroneous decoding, packet retransmission, i.e., ARQ, is performed with the aid of relays. This dramatically improves the diversity gain since the fading channels connecting the source and relays to the destination are independent. Cooperative relaying presents a potential alternative to classical ARQ and has recently received a lot of attention in the research community (see for instance [4], [5]).

In relay communications, different approaches can be used to relay the message from the source to the destination. The well known modes are amplify and forward (AF), and decode and forward (DF). In AF mode, the signal packet received from the source is simply amplified and transmitted to the destination. The DF mode involves more processing since the relay decodes then re-encodes the received data packet before relaying it to the destination. The performance of each mode mainly depends on the distances separating the relay from the source and destination. In general, the DF strategy suffers from error propagation [6], [7]. Selective DF overcomes this limitation by activating packet relaying only when a relay is able to correctly decode the data packet [8]. In the case of erroneous decoding at the relay, the incurring silence due to the inactivity of the relay to destination link reduces the overall system throughput. An alternative technique to alleviate this problem is to directly retransmit the packet from the source in the case of decoding failure at the relay [6], [9]. This approach can be implemented by exchanging positive/negative acknowledgment (ACK/NACK) messages in the network, and is refereed to as ACK/NACK-aided DF [10], [11].

Multiple-input-multiple-output (MIMO), where network elements are equipped with multiple transmit and receive antennas, will present a main building block in the next generation of beyond fourth generation (B4G) systems. MIMO techniques provide an enormous increase in the diversity gain and/or the multiplexing gain [12]. In practical wireless MIMO systems employing single carrier (SC) transmission, the communication link suffers from inter-symbol interference (ISI) caused by frequency selective fading.

In this paper, we focus on broadband relay communication protocols operating over frequency selective fading channels. Most of the work performed in the area of relay communications have been reported in the case of flat fading channels. We consider the so-called relaying Protocol II [13] as it is widely recognized as an efficient relaying technique for increasing the network throughput. In Protocol II, the source broadcasts the information message to the destination and the relays during the first slot, while the relays transmit to the destination during the following slots. We focus on cooperative ARQ where packet relaying/retransmission is activated only when the destination is unable to decode the data packet [14], [15]. We review some packet combining techniques we have recently proposed. In these combining mechanisms, the destination exploits the multiple packet copies received from the source and, eventually multiple relays, to perform combining in an iterative (turbo) fashion by exchanging soft information between the soft packet combiner and the softinput-soft-output (SISO) decoder. Turbo packet combining is performed in such a way to jointly cancel ISI corrupting relay- 
destination links, and eventually source-relay links if AF mode is considered.

The remainder of the paper is organized as follows. In Section II, we provide an overview of three main operating modes for cooperative ARQ networks. In Section III, we briefly describe the structure of the iterative turbo packet combining receiver for each relaying mode. Performance analysis is provided in Section IV. Finally, the paper is concluded in Section V.

\section{COOPERATIVE ARQ FOR BROADBAND MIMO RELAYING}

\section{A. Broadband Cooperative $A R Q$}

We consider a relay-based wireless communication network where a source equipped with $M_{\mathrm{S}}$ transmit antennas sends messages to a destination equipped with $M_{\mathrm{D}}$ receive antennas with the assistance of $K-1$ relay nodes denoted as $\mathrm{R}_{2}, \cdots, \mathrm{R}_{k}, \cdots, \mathrm{R}_{K}$. Each relay $\mathrm{R}_{k}$ has $M_{\mathrm{R}_{k}}$ transmit and receive antennas. Cyclic prefix (CP)-aided SC transmission is assumed for all network links, i.e., source-relay $S \rightarrow R_{k}$, source-destination $\mathrm{S} \rightarrow \mathrm{D}$, and relay-destination $\mathrm{R}_{k} \rightarrow \mathrm{D}$. Let $E_{\mathrm{SR}_{k}}, E_{\mathrm{SD}}$ and $E_{\mathrm{R}_{k} \mathrm{D}}$ denote the enregies of links $\mathrm{S} \rightarrow \mathrm{R}_{k}, \mathrm{~S} \rightarrow \mathrm{D}$, and $\mathrm{R}_{k} \rightarrow \mathrm{D}$, respectively. The MIMO fading channels corresponding to all network links experience frequency selective fading, and each link has its own channel memory. At the source, an information packet is first encoded then interleaved with the aid of a semi-random interleaver, then space-time multiplexed and mapped over the elements of signalling alphabet $\mathcal{Q}$. This results into a symbol matrix $\mathbf{S} \in \mathcal{Q}^{M_{\mathrm{S}} \times T}$ where $T$ is the number of channel use. During the first time slot, the source broadcasts matrix $\mathbf{S}$ to the destination and the $K-1$ relays. We suppose that the thermal noise variance is $\sigma^{2}$ and is identical for relays and the destination. In subsequent slots, relay nodes forward the received message to the destination depending on the decoding outcome. The relaying mechanism depends on the operation mode, and is detailed in the following subsections.

\section{B. AF Mode}

In the case of $A F$ mode, each relay node $R_{k}$ first removes the CP signal word from signals received from the source during the first slot. If relay $\mathrm{R}_{k}$ is requested to send during slot $k$, then it simply amplifies the signal obtained after the processing during the first slot, appends a CP signal word to it, and transmits the resulting signal to the destination. The energy of the equivalent $\mathrm{S} \rightarrow \mathrm{R}_{k} \rightarrow \mathrm{D}$ link is

$$
E_{k}=\frac{E_{\mathrm{R}_{k} \mathrm{D}} E_{\mathrm{SR}_{k}}}{M_{\mathrm{S}} E_{\mathrm{SR}_{k}}+\sigma^{2}}
$$

while the number of discrete taps is $L_{k}=L_{\mathrm{SR}_{k}}+L_{\mathrm{R}_{k} \mathrm{D}}-1$, where $L_{\mathrm{SR}_{k}}, E_{\mathrm{SR}_{k}}, L_{\mathrm{R}_{k} \mathrm{D}}$, and $E_{\mathrm{R}_{k} \mathrm{D}}$ denote the number of taps and the energy corresponding to links $S \rightarrow \mathrm{R}_{k}$ and $\mathrm{R}_{k} \rightarrow$ $\mathrm{D}$, respectively. Note that the multipath channel corresponding to the equivalent $\mathrm{S} \rightarrow \mathrm{R}_{k} \rightarrow \mathrm{D}$ link is the convolution of the channels of $S \rightarrow R_{k}$ and $R_{k} \rightarrow D$ links. Its expression can be found in [10].

\section{Selective DF Mode}

When the DF mode is considered, each relay node $\mathrm{R}_{k}$ first removes the CP signal word, then demodulates and decodes the information packet. When this relay is requested to transmit at slot $k$, it re-encodes and modulates the data block, appends a symbol CP word to symbol matrix $\mathbf{S}$, then transmits the resulting block to the destination. In selective $\mathrm{DF}$, the activation of relay $\mathrm{R}_{k}$ during slot $k$ depends on the decoding outcome at both relay $\mathrm{R}_{k}$ and the destination: If the destination is unable to decode the data packet at slot $k-1$, it requests a retransmission from relay $\mathrm{R}_{k}$ at slot $k$. The transmission link $\mathrm{R}_{k} \rightarrow \mathrm{D}$ is then activated at slot $k$ only if relay $\mathrm{R}_{k}$ was able to decode the data block received from the source during the first slot. Otherwise, the link is not activated and relay $\mathrm{R}_{k}$ keeps silent during slot $k$. The channel observed at slot $k$ is therefore the one corresponding to link $\mathrm{R}_{k} \rightarrow \mathrm{D}$, and consequently,

$$
E_{k}=E_{\mathrm{R}_{k} \mathrm{D}}, \text { and } L_{k}=L_{\mathrm{R}_{k} \mathrm{D}}
$$

\section{ACK/NACK-Aided DF Mode}

The ACK/NACK-aided DF mode prevents the throughput loss caused by possible "silence" periods that occur in the case of selective DF relaying. When a retransmission is requested at slot $k$, and relay $\mathrm{R}_{k}$ was unable to decode the data message during the first slot, the source directly retransmits the message to the destination during slot $k$. Therefore, the channel obeserved by the destination at slot $k$ depends on the decoding outcome at relay $\mathrm{R}_{k}$. It either corresponds to the channel of the $\mathrm{R}_{k} \rightarrow \mathrm{D}$ link if successful decoding is achieved by relay $\mathrm{R}_{k}$, or that of the link connecting the source and the destination at slot $k$ if the packet is erroneously decoded at relay $\mathrm{R}_{k}$. We get,

$$
\left\{\begin{array}{ll}
E_{k}=E_{\mathrm{R}_{k} \mathrm{D}}, L_{k}=L_{\mathrm{R}_{k} \mathrm{D}}, & \text { if succes. decod. } @ \mathrm{R}_{\mathrm{k}} \\
E_{k}=E_{\mathrm{SD}}, L_{k}=L_{\mathrm{SD}}, & \text { if unsucces. decod. } @ \mathrm{R}_{k}
\end{array} .\right.
$$

\section{Turbo PACKet COMBINING For BROADBAND COOPERATIVE ARQ}

\section{A. Brief Overview of the Concept}

The concept of turbo packet combining for broadband cooperative ARQ was introduced in [10], [11] as an efficient technique to combine packets received from multiple relays in a cooperative ARQ MIMO network. It presents an extension of the turbo packet combining methods initially proposed for conventional broadband ARQ systems [16], [17] to relay communications.

Let us suppose that a retransmission is required at slot $k$. Turbo packet combining is performed using signals received during $k$ consecutive slots. The receiver first deletes the $\mathrm{CP}$ signal word from the packet of signals received at each slot $k$, and constructs the discrete Fourier transform (DFT) of the resulting signal frame. Combining of signals is then performed in the frequency domain by iteratively exchanging soft loglikelihood ratio (LLR) values about coded and interleaved bits between the soft packet combiner and the SISO decoder. 


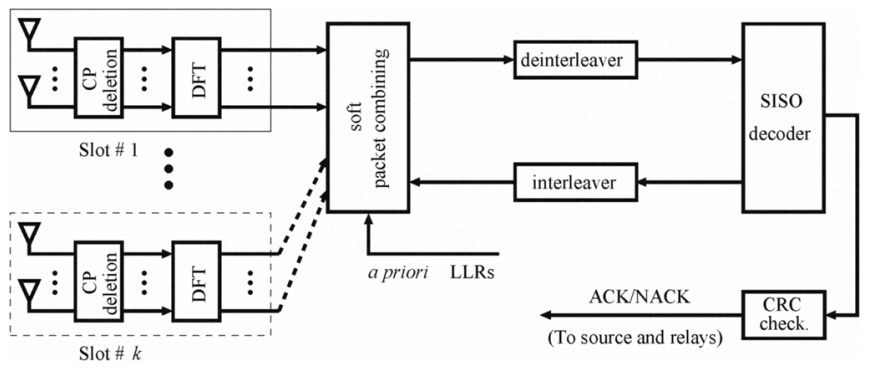

Figure 1. The block diagram of the turbo packet combining receiver scheme at slot $k$.

After a preset number of iterations, the decision about the data packet is performed. If the decoding is erroneous, a NACK message is sent to relay $k+1$ to start the packet retransmission process depending on the relaying scheme in use. If the decoding is successful, the destination broadcasts an ACK message to the source and relays to stop relaying and move on to the next information frame during the next slot. The block diagram of the iterative turbo packet combining receiver is depicted in Fig. 1. Details regarding the derivation of the turbo packet combiner can be found in [10], [11]. In the following, we briefly describe turbo packet combining for each relaying mode.

\section{B. Turbo Packet Combining for AF}

In the case of $\mathrm{AF}$, no processing is performed at the relays. Each relay only removes the $\mathrm{CP}$ word from the received signal packet, appends the $\mathrm{CP}$ word corresponding to transmission over the relay-destination link, amplifies then transmits the resulting signal block to the destination. At the destination receiver, turbo equalization is performed, in the frequency domain, jointly for all equivalent channels $S \rightarrow R_{2} \rightarrow D, \cdots$, $\mathrm{S} \rightarrow \mathrm{R}_{k} \rightarrow \mathrm{D}$ using the concept of virtual receive antennas and soft minimum mean square error (MMSE)-aided frequency domain equalization (FDE).

\section{Turbo Packet Combining for Selective DF}

In the case of selective $D F$, each relay $R_{k}$ first removes the CP word from the block of received signals then performs turbo MMSE FDE to iteratively decode the data packet received from the source. If the packet is correctly decoded, and $\mathrm{R}_{k}$ is requested to relay the information block during slot $k$, then it simply appends a $\mathrm{CP}$ symbol word to matrix $\mathbf{S}$ and transmits it to the destination. At the receiver side, turbo MMSE FDE is conducted to jointly equalize (i.e., combining and ISI cancellation) transmissions corresponding to slots $1, \cdots, k$.

\section{Turbo Packet Combining for ACK/NACK-Aided DF}

When ACK/NACK-aided DF is considered, turbo FDE is performed at the relays as in the case of selective DF. At the destination receiver, turbo packet combining employs MMSE FDE, and signals used in combining are either received from relays or the source depending on the ACK/NACK feedback at each slot. An optimized adaptive implementation algorithm can be found in [18].

\section{Performance Evaluation}

In this section, we provide block error rate (BLER) performance of turbo packet combining for broadband cooperative ARQ operating over MIMO fading channels. We consider a relay communication network where only one relay $R$ helps the source in relaying erroneously received packets to the destination. For the sake of simplicity, we suppose that the distances between the source and relay, i.e., $l_{\mathrm{SR}}$, relay and destination, i.e., $l_{\mathrm{RD}}$, and source and destination, i.e., $l_{\mathrm{SD}}$, are normalized in such a way that $l_{\mathrm{SR}}+l_{\mathrm{RD}}=l_{\mathrm{SD}}=1$. The frequency selective fading channels connecting the source, relay, and destination, i.e., $\mathrm{S} \rightarrow \mathrm{R}, \mathrm{R} \rightarrow \mathrm{D}$, and $\mathrm{S} \rightarrow \mathrm{D}$ have the same channel profile: $L_{S \mathrm{R}}=L_{\mathrm{RD}}=L_{\mathrm{SD}}=3$, and equal power taps with a path loss exponent $\kappa=3$. The link average energies are therefore, $E_{\mathrm{SR}}=l_{\mathrm{SR}}^{-\kappa}, E_{\mathrm{RD}}=l_{\mathrm{RD}}^{-\kappa}$, and $E_{\mathrm{SD}}=l_{\mathrm{SD}}^{-\kappa}$. The CP length for all links is $T_{C P}=3$. The space-time transmitter at the source node is made of a 16 state convolutional encoder with polynomial generators $(35,23)_{8}$, and quadrature phase shift keying (QPSK) modulation. The length of the code frame is 2048 bits including tail bits. The iterative turbo MMSE FDE receiver runs three turbo iterations. We use conventional LLR-level based packet combining to evaluate the gain offered by MMSE FDE-aided turbo packet combining. In LLR-level combining strategy, the turbo equalization is performed separately for each slot, extrinsic LLRs generated by the soft demapper are simply added together with those obtained at the last iteration of previous slots before SISO decoding. We also use the outage probability to evaluate the diversity gain achieved by both combining techniques. For all curves, the signal to noise ratio (SNR) in the abscissa axis denotes the SNR of the $\mathrm{S} \rightarrow \mathrm{D}$ link per symbol per receive antenna.

In Fig. 2, we provide BLER performance for a relaying system with the same number of transmit and receive antennas $M_{\mathrm{S}}=M_{\mathrm{R}}=M_{\mathrm{D}}=2$, and a normalized source to relay distance $l_{\mathrm{SR}}=0.3$. We observe that the MMSE FDE aided turbo packet combining clearly outperforms LLR-level packet combining. The gap with the outage performance at $10^{-2}$ BLER is less than $1 \mathrm{~dB}$ for all cooperative ARQ modes: AF, selective DF, and ACK/NACK-aided DF. From the slopes of BLER curves, we notice that both turbo combining strategies fail to achieve the diversity order of the cooperative ARQ relaying system.

In Fig. 3, we report the BLER performance for a onerelay cooperative ARQ network where the source and relay are equipped with two antennas, i.e., $M_{\mathrm{S}}=M_{\mathrm{R}}=2$, while the destination has only one receive antenna, i.e., $M_{\mathrm{D}}=1$. The normalized distance between the source and relay is increased to $l_{\mathrm{SR}}=0.7$. In this case, MMSE FDE turbo packet combining significantly outperforms LLR-level combining. For instance, the performance gap between the two techniques is more than $2 \mathrm{~dB}$ at $10^{-2}$ BLER. MMSE FDE manifests itself in almost achieving the diversity gain as it can be seen from the slope of the outage performance curve, while LLR-level has a limited diversity gain. 


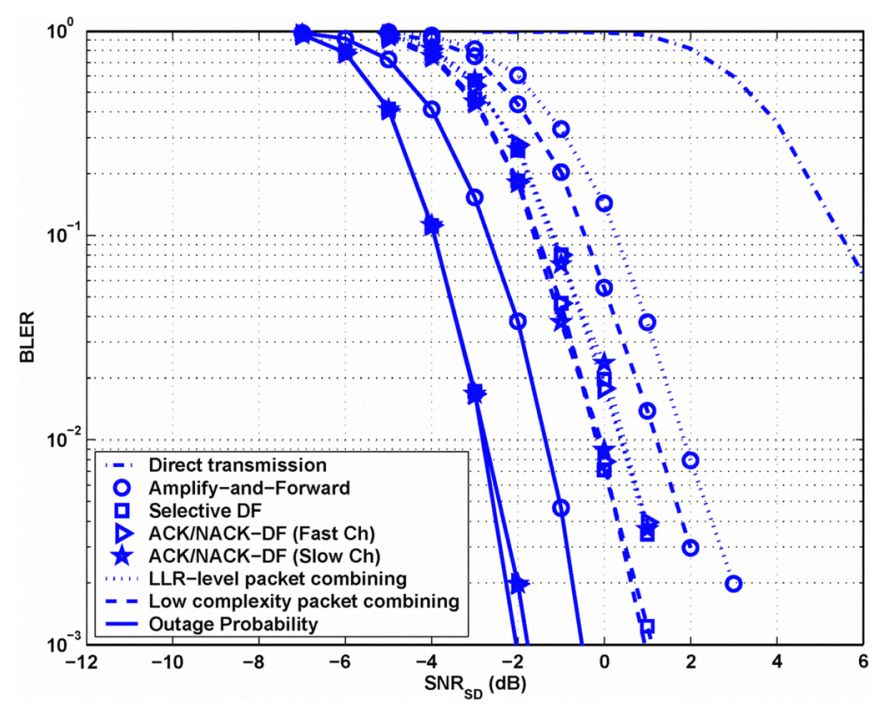

Figure 2. BLER performance for $M_{\mathrm{S}}=M_{\mathrm{R}}=M_{\mathrm{D}}=2$, and $l_{S R}=0.3$.

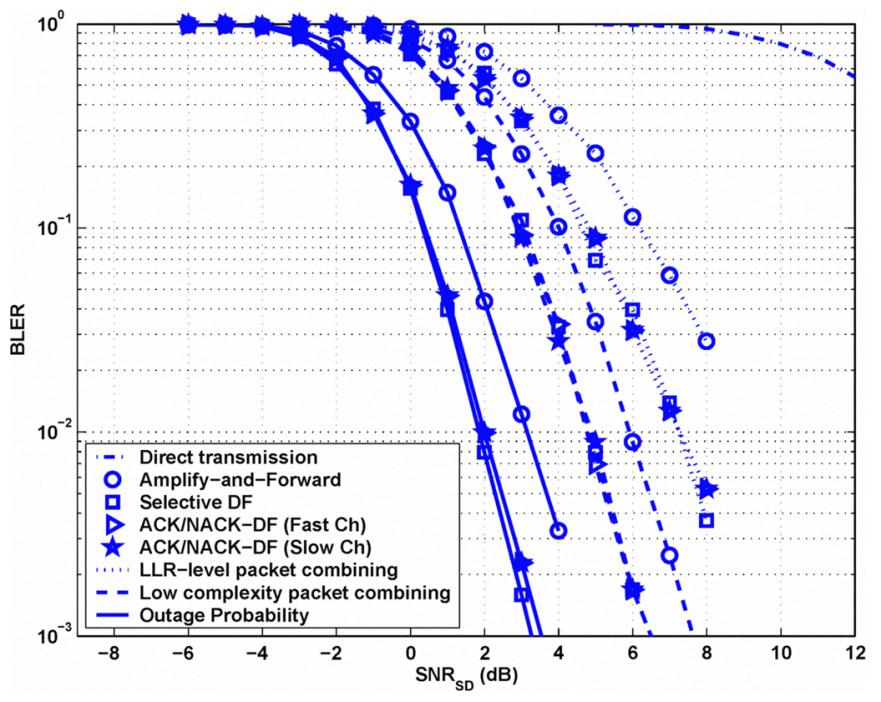

Figure 3. BLER performance for $M_{\mathrm{S}}=M_{\mathrm{R}}=2, M_{\mathrm{D}}=1$, and $l_{S R}=$ 0.7 .

\section{CONCLUSION}

In this paper, we provided an overview of some turbo packet combining techniques we have recently introduced for broadband cooperative ARQ systems. The combining techniques are based on turbo MMSE FDE methods. We examined their BLER performances in the case of AF, selective DF, and ACK/NACK-aided DF modes, and showed that they clearly outperform conventional LLR-level combining techniques.

\section{REFERENCES}

[1] S. Lin, D. J. Costello, and M. J. Miller, "Automatic repeat-request error control schemes," IEEE Communications Magazine, vol. 12, pp. 5-17, Dec. 1984.

[2] G. Caire, and D. Tuninetti, "ARQ protocols for the Gaussian collision channel," IEEE Trans. Inf. Theory, vol. 47, no. 4, pp. 1971-1988, Jul. 2001.

[3] E. Zimmermann, P. Herhold, and G. Fettweis, "The impact of cooperation on diversity-exploiting protocols," IEEE VTC-spring, Milan, Italy, May 2004.
[4] J. N. Laneman, G. W. Wornell, and D. N. C. Tse, "An efficient protocol for realizing cooperative diversity in wireless networks," IEEE International Symposium on Information Theory (ISIT), Washington, DC, June 2001.

[5] A. Sendonaris, E. Erkip, and B. Aazhang, "User cooperation diversity Part I \& Part II," IEEE Trans. Commun., vol. 51, pp. 1927-1948, Nov. 2003.

[6] J. N. Laneman, D. Tse, and G. W. Wornell, "Cooperative diversity in wireless networks: Efficient protocols and outage behavior," IEEE Trans. Inform. Theory, vol. 50, no. 12, pp. 3062-3080, Dec. 2004.

[7] J. Boyer, D. D. Falconer, and H. Yanikomeroglu, "Multihop diversity in wireless relaying channels," IEEE Trans. on Comm., vol. 52, pp. 18201830, Oct. 2004.

[8] F. Atay Onat, H. Yanikomeroglu, and S. Periyalwar, "Relay-assisted spatial multiplexing in wireless fixed relay networks,", IEEE GLOBECOM, San Francisco, USA, Nov.- Dec. 2006.

[9] G. Yu, Z. Zhang, and P. Qiu, "Cooperative ARQ in wireless networks: Protocols description and performance analysis", IEEE International Conference on Communications (ICC), Istanbul, Turkey, June 2006.

[10] H. Chafnaji, T. Ait-Idir, H. Yanikomeroglu, and S. Saoudi, "Joint turbo equalization for relaying schemes over frequency-selective fading channels," in Proc., ACM Intern. Wireless Commun. Mobile Comput. Conf. IWCMC 2009, Leipzig, Germany, Jun. 2009.

[11] H. Chafnaji, T. Ait-Idir, H. Yanikomeroglu, and S. Saoudi, "Turbo Packet Combining for Relaying Schemes over Multi-Antenna Broadband Channels," Submitted, IEEE Intern. Conf. Commun., ICC 2010, Cape Town, South Africa, May 2010.

[12] P. W. Wolniansky, G. J. Foschini, and R. A. Valenzuela, "V-BLAST : an architecture for realizing very high data rates over the rich scattering wireless channel," in Proc. Int. Symp. Signals, Systems, Electron., Pisa, Italy, Sep. 1998.

[13] R. U. Nabar, F. W. Kneubiihler, and H. Boelcskei, "Performance limits of amplify-and-forward based fading relay channels", IEEE International Conference on Acoustics, Speech, and Signal Processing (ICASSP), Montreal, Canada, May 2004.

[14] Y. Zhang, H. H. Chen, and M. Guizani, Cooperative Wireless Communications, Auerbach Publications, 2009.

[15] B. Zhao and M. C. Valenti, "Practical relay networks: A generalization of hybrid-ARQ," IEEE J. Select. Areas. Comm., vol. 23, no. 1, Jan. 2005.

[16] T. Ait-Idir, and S. Saoudi, "Turbo packet combining strategies for the MIMO-ISI ARQ channel," IEEE Trans. Commun., In Press.

[17] T. Ait-Idir, H. Chafnaji, and S. Saoudi, "Turbo packet combining for broadband space-time BICM ARQ systems with co-channel interference," Conditionally accepted, IEEE Trans. Wireless Commun.

[18] H. Chafnaji, T. Ait-Idir, H. Yanikomeroglu, and S. Saoudi, "On the design of turbo packet combining schemes for relay-assisted systems over multi-antenna broadband channels, " Submitted, IEEE 71st SemiAnnual Vehic. Technol. Conf., VTC10Spring, Taipei, Taiwan, May 2010. 